\title{
STUDY ON WATER ENTRY PROBLEM USING A LEVEL-SET IMMERSED BOUNDARY METHOD*
}

\author{
WEI BAI \\ Department of Civil and Environmental Engineering, National University of Singapore, \\ Block E1A, \#07-03, 1 Engineering Drive 2, Singapore 117576, Singapore \\ w.bai@nus.edu.sg \\ CHENGZHONG HUO \\ Department of Civil and Environmental Engineering, National University of Singapore, \\ Block E1A, \#07-03, 1 Engineering Drive 2, Singapore 117576, Singapore \\ ceehc@nus.edu.sg
}

Published 17 November 2014

\begin{abstract}
Water entry of a solid through the free surface is a persisting field of research in ship hydrodynamics applications. Indeed, the knowledge of pressure forces acting on structures is necessary to ensure the verification of safety criteria in the design and operation. However, in water entry problems, jets can be generated, thus an effective numerical model is needed to capture this complicated breaking water surface. In this paper, the level set method is adopted, which has been shown to be capable of capturing interface evolution when the topological change of shape is extremely large, or merging, breaking and pinching occur. Moreover, the incorporation of an immersed boundary method with this free surface capture scheme implemented in a NavierStokes solver allows the interaction between fluid flow with free surface and moving bodies of almost arbitrary shape to be modeled. The developed Level-Set Immersed Boundary Method is applied to simulate the water entry of a rectangular body with different velocities into the still water. The complicated surface profile, velocity field and pressure are obtained. The simulation is also carried out for the same body exiting the water, and some preliminary results are presented.
\end{abstract}

Keywords: Level set method; immersed boundary method; water entry; free surface capturing; moving body.

\section{Introduction}

Offshore installation operations are applicable to all structures situated offshore, as most of them are built onshore, then transported and installed at the operating site. As one of the most common method of offshore installation, the offshore lift installation always requires the understanding on the water entry problem, in which high water impact pressure can be induced and it further affects the tension in the crane wires. When the

\footnotetext{
* This is an Open Access article published by World Scientific Publishing Company. It is distributed under the terms of the Creative Commons Attribution 3.0 (CC-BY) License. Further distribution of this work is permitted, provided the original work is properly cited.
} 
structure impacts the free surface, a flow singularity occurs due to a high pressure peak localized at the spray root which makes the water entry and exit problem more complicated and difficult. Implementation of deforming and moving boundaries within fluid solvers presents possibilities for the numerical analysis of this fluid-structure interaction. However, it is difficult to simulate the irregular objects undergoing large motions and complex free surface evolutions, such as the water entry problem discussed here.

In this paper, we do not focus on the boundary-fitted method. Instead, the immersed boundary method, a non-boundary conforming method, is implemented to simulate a moving body in the fluid domain. The immersed boundary method, which was firstly proposed by Peskin ${ }^{1}$ in 1972, enables to represent a body of any shape within a flow field by adding a forcing term to the governing equations, without the necessity of performing the mapping procedures. According to the review paper of Mittal et $a l_{.}{ }^{2}$, the resulting fundamental dichotomy in immersed boundary methods includes the continuous forcing approach and the discrete forcing approach. In the first approach, a forcing function is explicitly added to the continuous governing equations prior to the dicretization of them. The original method of Peskin ${ }^{1}$ is a typical example. However, this technique may induce high-frequency spurious oscillations and restrict the computational time step, which makes the simulation of flow fields in complex domains very expensive. In the second approach, the forcing term is either explicitly or implicitly introduced after the NavierStocks equations being discretized ${ }^{3-5}$. Compared with the first approach, the direct forcing methods have the advantage of allowing a sharper representation of the immersed boundary.

The present immersed boundary method is based on the direct forcing technique proposed by Fadlun et al. ${ }^{4}$. It has the advantage of simplifying the grid generation and its inherent simplicity to study moving body on fixed Cartesian grids. The key concept is that the velocity of the fluid must equal to the velocity of the moving body at its surface. This implies that introduction of a body-force to the momentum equations at certain points in the flow can simulate the effect of the investigated body in the flow. This idea enables the immersed boundary method to mimic the complex body through a suitably introduced artificial force adding to the momentum. In this paper, the immersed boundary method is implemented in a well-developed free surface flow solver. In this flow solver, the arbitrary free surface evolutions can be captured by using the level set method. By using the present numerical model, some preliminary results are obtained for the water entry of a rectangular box with different specified velocities, as well as its exit from the still water.

\section{Mathematical Model}

\subsection{Governing equations}

In the $2 \mathrm{D}$ wave-structure interaction, the flow of an incompressible viscous fluid is governed by the Navier-Stokes equations 


$$
\frac{\partial u_{i}}{\partial t}+u_{j} \frac{\partial u_{i}}{\partial x_{j}}=\frac{1}{\rho(\phi)}\left(-\frac{\partial p}{\partial x_{i}}+\frac{\partial \tau_{i j}}{\partial x_{j}}\right)+f_{i},
$$

and the continuity equation

$$
\frac{\partial u_{i}}{\partial x_{i}}=0
$$

where Cartesian tensor notation is used, ( $i=1$ and 2$), u_{j}, p$ and $x_{j}$ are the velocities, pressure and spatial coordinates respectively. $f_{i}$ represents the momentum forcing components, $\rho$ is the fluid density and $\tau_{i j}$ are the viscous stress components given by

$$
\tau_{i j}=\mu(\phi)\left(\frac{\partial u_{i}}{\partial x_{j}}+\frac{\partial u_{j}}{\partial x_{i}}\right)
$$

where $\mu$ is the fluid viscosity.

\subsection{Free surface equations}

We investigate the motion of two incompressible fluids and track the movement of the free surface implicitly by the Level Set Method (LSM). In LSM a scalar quantity $\varphi$, known as the level set function, is specified throughout the domain to represent the location of grid cells relative to the surface. Here, we define $\varphi$ to be a signed distance function, which measures the shortest distance from the grid cell to the free surface (i.e. $|\nabla \varphi|=1)$ and is positive in one fluid phase and negative in the other. The evolution of the level set function $\varphi$ is governed by

$$
\frac{\partial \phi}{\partial t}+u_{i} \frac{\partial \phi}{\partial x_{i}}=0
$$

In the governing equations, both $\rho$ and $\mu$ vary dependent on the local fluid phase properties. If $\rho$ and $\mu$ are discontinuous there, the solution of the Navier-Stokes equations will yield instabilities at the interface. In order to avoid numerical instabilities caused by the sharp gradients present, $\rho$ and $\mu$ are smoothed over a small distance $\varepsilon=2 \Delta x$ across the surface by use of a Heaviside function $H$. We calculate $\rho$ and $\mu$ by

$$
\begin{aligned}
& \rho(\phi)=\rho_{\text {air }}+H(\phi)\left(\rho_{\text {water }}-\rho_{\text {air }}\right), \\
& \mu(\phi)=\mu_{\text {air }}+H(\phi)\left(\mu_{\text {water }}-\mu_{\text {air }}\right),
\end{aligned}
$$

where

$$
H(\phi)=\left\{\begin{array}{l}
0 \\
\frac{1}{2}+\frac{\phi}{2 \varepsilon}+\frac{1}{2 \pi}\left(\frac{\pi \phi}{\varepsilon}\right) . \\
1
\end{array}\right.
$$


At present, we neglect the influence of surface tension since we are currently interested in the large scale dynamics of gravity waves.

\section{Numerical Approach}

\subsection{Navier-Stokes solver}

The Navier-Stokes equations are discretized using a finite difference method on a staggered grid, in which the velocity components are defined at centers of cell faces in the $x, y$ and $z$ directions, respectively, and all other variables, i.e. $p, \varphi, \rho$ and $\mu$ defined at grid cell centers. We discretize the temporal gradient with a second order Runge-Kutta Total Variation Diminishing (RK-TVD) scheme. The governing Equations (1) and (2) are discretized using a fractional step method

$$
\begin{gathered}
u_{i}^{* *}=u_{i}^{n}+\Delta t\left(\frac{1}{\rho^{n}(\phi)}\left(\frac{\partial \tau_{i j}^{n}}{\partial x_{j}}\right)-u_{j}^{n} \frac{\partial u_{i}^{n}}{\partial x_{j}}+g_{i}+f_{i}\right), \\
\frac{\partial}{\partial x_{i}}\left(\frac{1}{\rho^{n}(\phi)} \frac{\partial p^{n}}{\partial x_{i}}\right)=\frac{1}{\Delta t}\left(\frac{\partial u_{i}^{* *}}{\partial x_{i}}\right), \\
\hat{u}_{i}^{n+1}=u_{i}^{* *}-\frac{\Delta t}{\rho^{n}(\phi)} \frac{\partial p^{n}}{\partial x_{i}}
\end{gathered}
$$

where $u^{* *}$ is the predicted velocity. $f_{i}$ is the momentum forcing used to enforce the desired boundary conditions on an immersed boundary interface and $\Delta t$ is the time step. The solution of Eq. (8) is achieved with second order central differencing to calculate the spatial gradients. The gradient of the computed pressure field is added to Eq. (9) to give a prediction of the new velocity field.

In discretizing the convective term in Eq. (1) it is essential to avoid the introduction of numerical instabilities due to the sharp density gradient at the surface. We employ first order upwinding to ensure stability. The diffusive term in Eq. (1) is discretized with a second order central difference. The time step is restricted by the CFL condition and gravity as discussed in Ref. 6.

\subsection{Free surface solver}

Accurate solution of the level set equation (Eq. 4) is crucial to capture the correct surface physics. Here, we discretize $\varphi$ at cell centers and calculate velocity gradients with a fifth order HJ-WENO scheme ${ }^{7}$. Temporal gradients are resolved with a third order RK-TVD scheme. Since only the location of the surface is of interest, $\varphi$ can be solved in a narrow band close to the interface. For the HJ-WENO scheme the narrow band occupies six cell widths either side of the surface in all directions ${ }^{8}$. As $\varphi$ is evolved in time it may deviate away from being a signed distance function (i.e. $|\nabla \varphi| \neq 1$ ) requiring reinitialisation. Here, 
we reinitialize $\varphi$ with an efficient fast marching technique at every time step, see Ref. 9 for details.

\section{Immersed Boundary Treatment}

Fluid flow over a body can exert a force on the no-slip surface and will, in turn, apply a force with the magnitude and direction opposing to the local flow. As a result, the fluid flow can be brought to rest on the body surface ${ }^{10}$. Imposition of nodal force at the immersed boundary is the key issue and also the main difficulty in developing an immersed boundary method.

\subsection{Identification of forcing points}

Obviously, it is necessary to have the detailed information about the immersed boundary in order to locate points which lie near the interface. To approximately represent the immersed boundary, a series of forcing points should be identified, on which the momentum forces act. For a node to be identified as a forcing point, it must lie in the solid phase and have at least one neighboring point in the fluid phase, as performed in Ref. 5. In our program, for any segment of boundary interface, we first look at which quadrant it belongs to, based on the angle. In each quadrant, we further compare the angle of the segment with the diagonal line. Larger and smaller than the diagonal lines, we have different treatments to search the forcing force from the different directions.

\subsection{Determination of the imposed force term}

It is noted that the forcing term $f_{i}$ must be determined prior to the computation of the predicted velocities $u^{* *}$. This term is prescribed at each time step to establish the desired boundary velocity $V_{i b}$. For a time-stepping scheme, this force can be expressed as ${ }^{11}$

$$
\frac{u_{i}^{n+1}-u_{i}^{n}}{\Delta t}=R S H_{i}+f_{i},
$$

where $R H S_{i}$ includes the convective, viscous, pressure gradient and body force of the governing equations. When the grid line coincides with the immersed boundary that means the forcing $f_{i}$ must yield $u_{i}^{n+1}=V_{i b}{ }^{n+1}$ on the immersed boundary, where $V_{i b}{ }^{n+1}$ is the specified velocity along the immersed boundary, the forcing is given from the above equation,

$$
f_{i}=\frac{V_{i b}-u_{i}^{n}}{\Delta t}-R S H_{i} .
$$

This forcing is direct in the sense that the desired boundary condition can be satisfied at every time step but only holds when the immersed boundary coincides with the grid. In general, Eulerian grid is not required to conform to the immersed boundary geometry in practical applications. The prescribed momentum forcing term will act only on the points 
nearest the immersed boundary. Therefore, $f_{i}$ needs to be computed at grid points near and not exactly on the interface. The value, $u_{f}$, at the forcing points is not known and has to be reconstructed using the information from the interface and surrounding field. Therefore, the force on the forcing points can be expressed as

$$
f_{i}=\frac{u_{f}-u_{i}^{n}}{\Delta t}-R S H_{i} .
$$

Evaluating the momentum force $f_{i}$ requires essentially no additional CPU time since there are no additional terms to compute. Nor does it influence the stability of the time advancement scheme.

\subsection{Interpolation technique}

The interpolation method used to calculate the enforced velocity at the forcing point has been adapted based on the method described in Ref. 12. This method requires a point on the immersed boundary which possesses a normal that passed through the forcing point to be identified for every forcing point, as shown in Fig. 1. First a virtual point, $u_{v}$, of the forcing point, $u_{f}$, is located along the normal to the boundary. The location of the virtual point on the line normal to the boundary is determined in such a way that $d_{1}=d_{2}$, where $d_{1}$ is the distance between the forcing point and perpendicular point, $u_{b}$, and $d_{2}$ is the distance between the perpendicular point and virtual point. If any of the points in the stencil 1-4 is a forcing point, the virtual point is relocated in a way such that $d_{2}>d_{1}$. This can be done by increasing the initial distance by one grid distance. Then the value of the virtual point $u_{v}$ can be interpolated from the surrounding grid points 1-4 using bilinear interpolation. And then based on $u_{v}$, and the boundary value $u_{b}$, we can extrapolate the value $u_{f}$ on the forcing point. Based on the obtained $u_{f}$, we can calculate the force $f_{i}$ using Eq. (12).

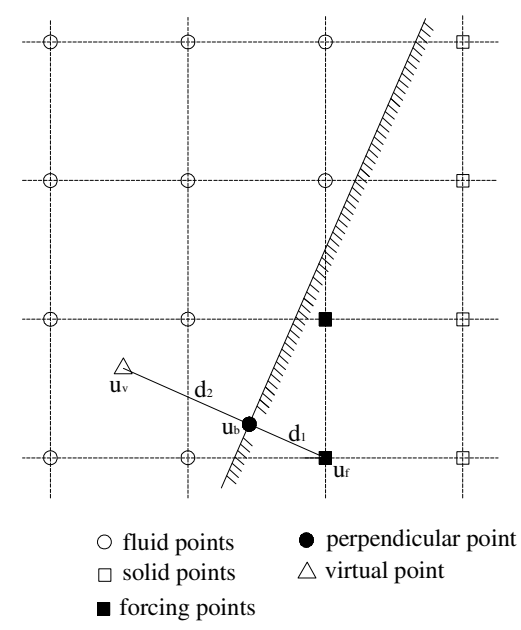

Fig. 1. Schematic of interpolation scheme. 


\section{Results}

To demonstrate the capability of the proposed LS-IBM for the water entry problem, we consider a computational domain of $2 L \times 2 L$ and the calm water depth is $1.6 L$, where $L=$ $1 \mathrm{~m}$. A square obstacle with geometry $h \times h$, where $h=0.31 \mathrm{~m}$, is located at the middle of the tank. The initial height of the obstacle above the water surface is $0.02 \mathrm{~m}$. A schematic view of the problem is shown in Fig. 2.

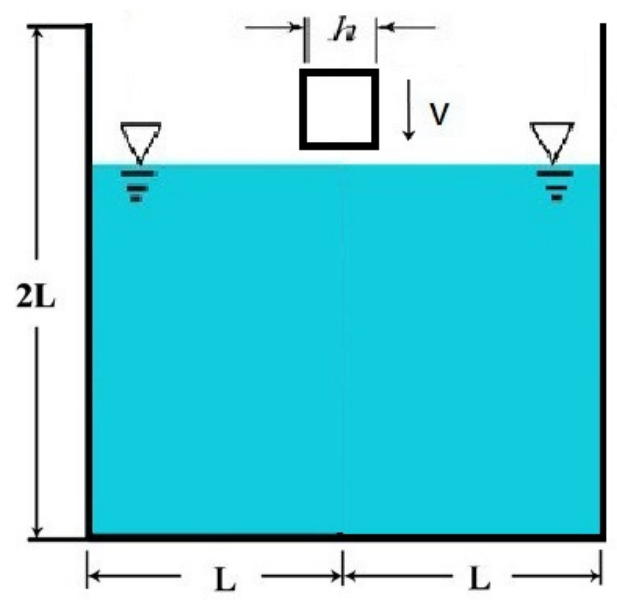

Fig. 2. Geometry and definition of the water entry problem.

The first case we considered is that the square obstacle starts its downward motion with a constant velocity $V=0.5 \mathrm{~m} / \mathrm{s}$. Snapshots at several time instants are shown in Fig. 3. As the square obstacle enters the free surface, it deforms the free surface and jets are generated at both sides of the obstacle in the entry process. When the body moves deeper, it tends to be submerged by the water, and the water from the left-hand and right-hand sides will merge together, forming a "mushroom"-type surge wave. The surge wave will further split into two parts, and each of them will propagate towards one direction independently. For the purpose of demonstration, the pressure field at $t=1.0$ is also presented in Fig. 4. The pressure distribution seems to be symmetric and very regular.

Furthermore, we also compare the water surface profiles at several time instants for the water entry of a rectangular obstacle with three different velocities in Fig. 5. When the body is moving with a larger velocity at $V=1.0$, the flow pattern is similar to that at $V=0.5$. As the higher speed of the body can cause a more violent water splash, therefore, when $V=1.0$ the water from the two sides will meet later than that for $V=0.5$. However, if the speed of the body is as high as $V=2.0$, the wave evolution will be completely different. Because the body is moving very fast, we can notice a larger water splash remains there during the whole process. At the same time, a large air cavity is formed behind the body, and the water tends to fill in this air cavity at the end of our simulation. 

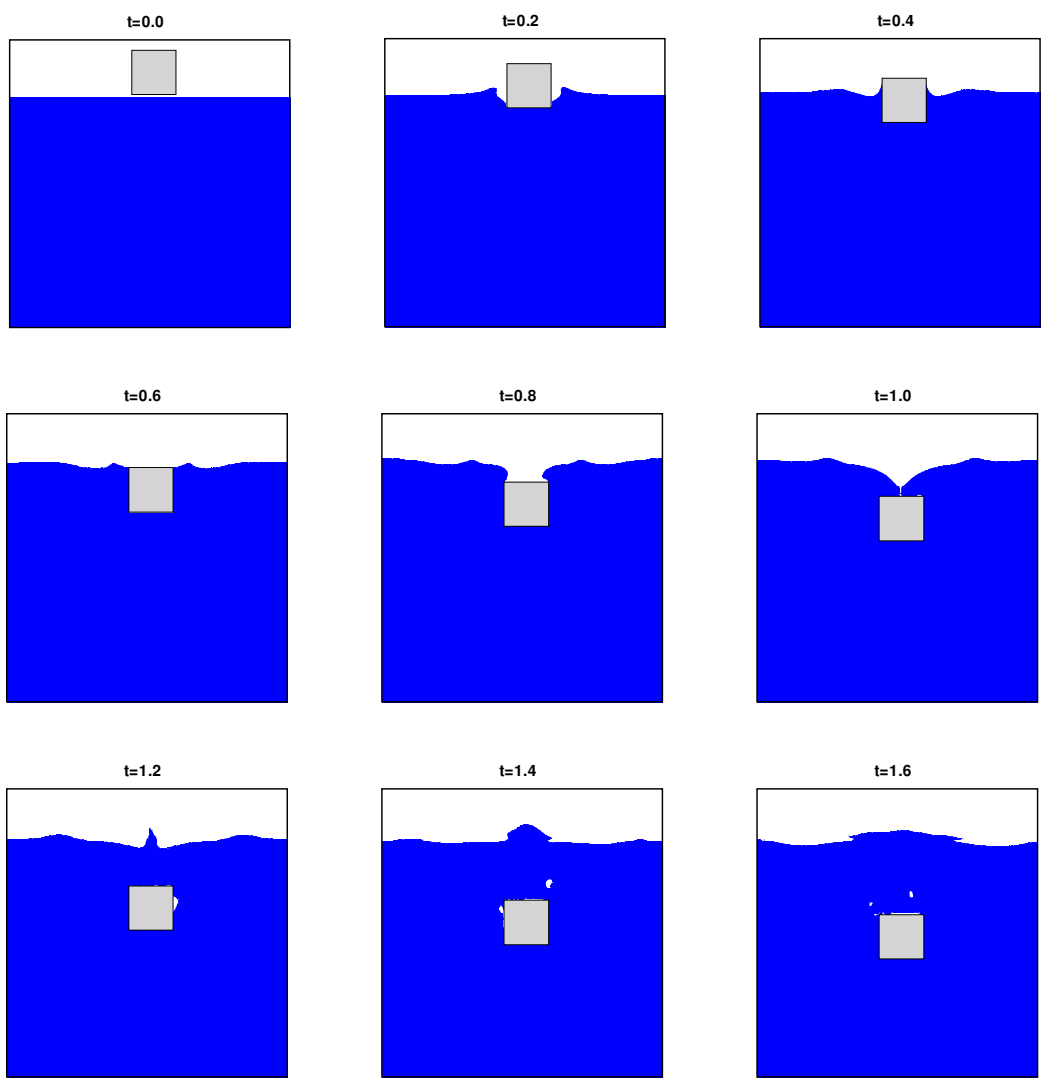

Fig. 3. Water profiles at several time instants for water entry of a square obstacle.

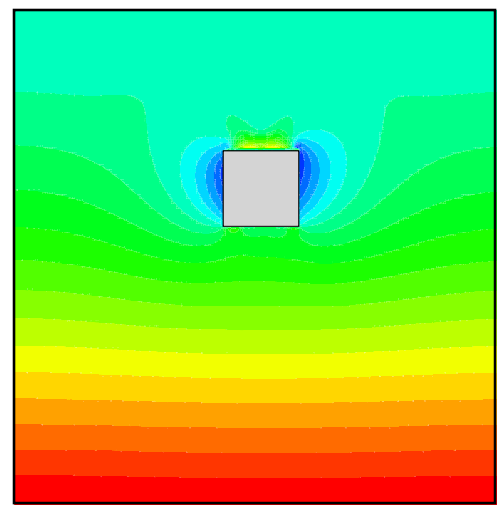

Fig. 4. Pressure field for water entry of a square obstacle at $t=1.0$. 
$t=0.2$

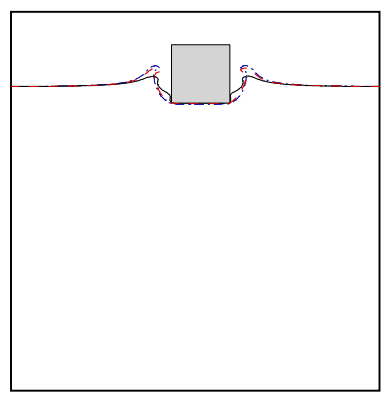

$t=0.8$

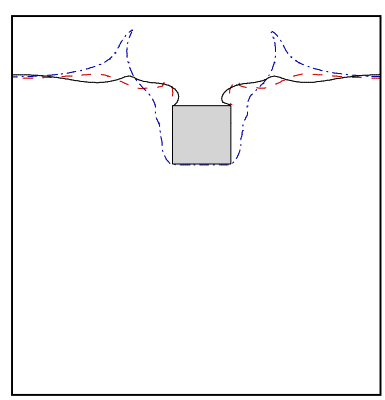

$t=1.2$

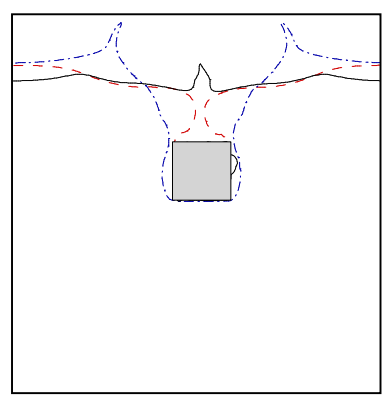

$\mathbf{t}=\mathbf{0 . 4}$

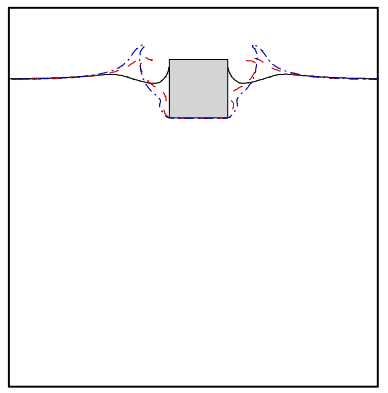

$\mathbf{t}=\mathbf{1 . 0}$

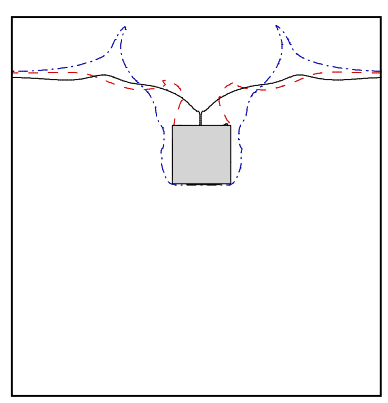

$t=1.6$

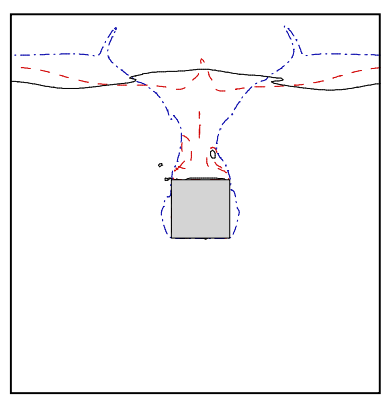

Fig. 5. Comparison of water profiles at several time instants for water entry of a square obstacle with three different velocities, 0.5 (solid line), 1.0 (dashed line) and 2.0 (dash-dot line).

Finally, the water exit problem is also investigated. In this case, the calm water depth is reduced to $1.0 \mathrm{~L}$. The initial distance from the obstacle to the water surface is $0.37 \mathrm{~m}$, and the obstacle is fully submerged in the water. The speed of the obstacle is fixed at $V=$ $0.5 \mathrm{~m} / \mathrm{s}$. Fig. 6 shows the distribution of the horizontal and vertical velocities at $t=0.6$. In addition, snapshots at several time instants are presented in Fig. 7, from which we can see the results predicted by the present numerical model seem to be reasonable and acceptable. 


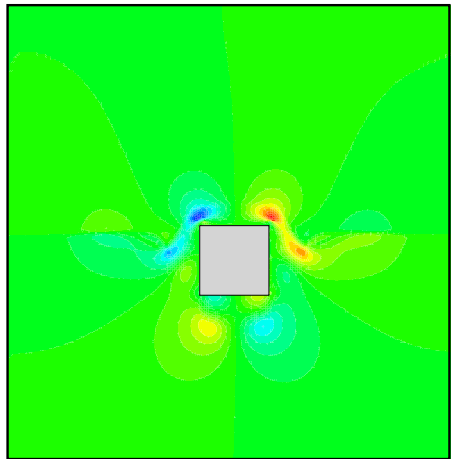

(a)

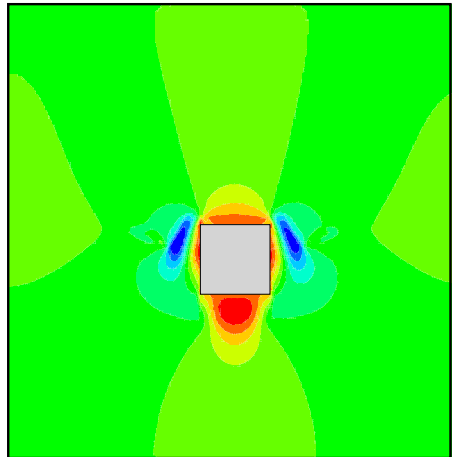

(b)

Fig. 6. Velocity field for water exit of a square obstacle at $t=0.6$ : (a) horizontal velocity and (b) vertical velocity.

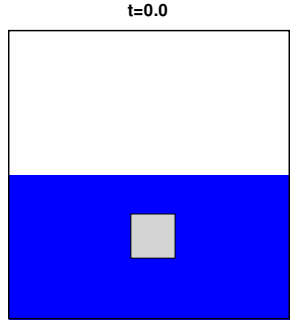

$\mathrm{t}=0.8$

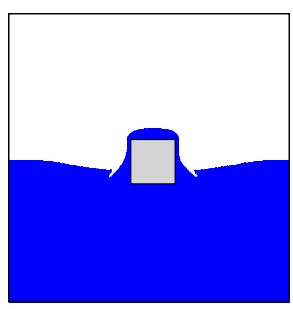

$\mathrm{t}=0.2$

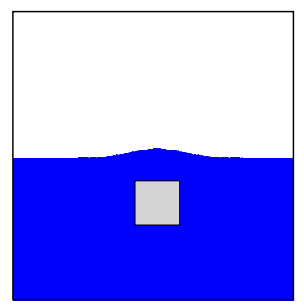

$t=1.2$

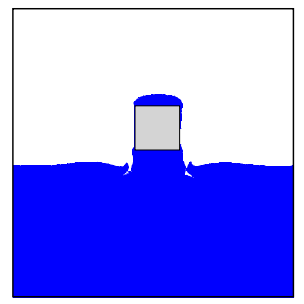

$t=0.6$

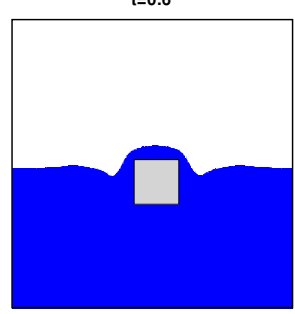

$t=1.4$

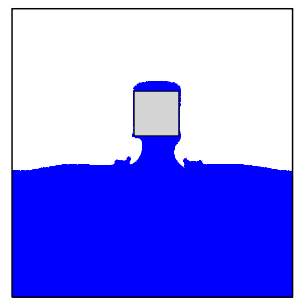

Fig. 7. Water profiles at several time instants for water exit of a square obstacle.

\section{Conclusions}

A numerical model based on the immersed boundary method is developed in this paper to simulate a body moving in the fluid domain. The level set method, in conjunction with the two-phase flow model adopted here enables the simulation of the fluid flow undergoing complicated deformation. The developed numerical model is successfully implemented to study the water entry and water exit problems, which indicates its capability of simulating the arbitrary body moving in a complicated free surface flow. The water entry of a rectangular obstacle with different constant speeds is investigated. 
The future work includes the comparison of the numerical results with more published experimental data, in order to have a complete validation for the present numerical model. To investigate various engineering problems is also part of our work plan.

\section{Acknowledgments}

The authors gratefully acknowledge the financial support provided by the Faculty Research Committee (FRC) Grant R-302-501-019-112.

\section{References}

1. C. S. Peskin, J. Comput. Phys. 10, 252 (1972).

2. R. Mittal and G. Iaccarino, Annual Review of Fluid Mechanics 37, 239 (2005).

3. J. Mohd-Yusof, Combined immersed boundary/B-spline method for simulations of flows in complex geometries, in Center Annual Research Briefs, NASA Ames/Stanford University, p. 317 (1997).

4. E. A. Fadlun, R. Verzicco, P. Orlandi and J. Mohd-Yusof, J. Comput. Phys. 61, 35 (2000).

5. J. Kim, D. Kim and H. Choi, J. Comput. Phys. 171, 132 (2001).

6. M. Kang, R. P. Fedkiw and X. D. Liu, J. Sci. Comput. 15, 323 (2000).

7. G. S. Jiang and D. Peng, SIAM J. Sci. Comput. 21(6), 2216 (2000).

8. D. Peng, B. Merriman, S. Osher, H. Zhao and M. Kang, J. Comput. Phys. 155, 410 (1999).

9. J. A. Sethian, A fast marching level set method for monotonically advancing fronts, in Proceedings of the National Academy of Science USA, 93, 1591 (1996).

10. D. Goldstein, R. Haandler and L. Sirovich, J. Comput. Phys. 105, 336 (1993).

11. E. Balaras, Computers and fluids 33, 375 (2004).

12. Y. Zhang, Q. Zou, D. Greaves, D. Reeve, H. R. Alison, D. Graham, J. Phil and X. Lv, Commun. Comput. Phys. 8(2), 265 (2010). 